\title{
Enhanced Unblocking From Sustained Post-Trial Surprise
}

\author{
Mark Haselgrove \\ The University of Nottingham
}

\author{
Shu K. E. Tam \\ The University of Oxford
}

\author{
Peter M. Jones \\ Staffordshire University
}

\begin{abstract}
Two appetitive conditioning experiments with rats investigated the mechanisms and properties of unblocking that results from the surprising omission of an expected post-trial unconditioned stimulus (US). Experiment 1 demonstrated unblocking under circumstances in which differences in the contribution of generalization decrement and within-compound associations are equated across experimental and control groups. Following Stage 1 training in which a conditioned stimulus (CS) A was followed by a US and a post-trial US, in Experiment 2 we arranged for the post-trial US to be present on some trials with AX but not others. Under these circumstances an enhancement of unblocking to $\mathrm{X}$ was observed, relative to a group who received standard unblocking by US omission. The implications of these results for attentional and US-processing theories of associative learning are discussed.
\end{abstract}

Keywords: blocking, unblocking, attention, associability, surprise

In a now classic series of experiments, Kamin $(1968,1969)$ gave rats paired presentations of a compound of two conditioned stimuli (CS) with an unconditioned stimulus (US): AX+. Conditioning to $\mathrm{X}$ was blocked if $\mathrm{A}$ had been paired with the US in a previous stage of the experiment: $\mathrm{A}+$. This blocking effect is a robust phenomenon in studies of conditioning and associative learning, having been demonstrated across a wide variety of species and behavioral procedures (e.g., Blaser, Couvillon, \& Bitterman, 2004; Dopson, Pearce, \& Haselgrove, 2009; Haselgrove \& Evans, 2010; Prados et al., 2012; Tennant \& Bitterman, 1975). Kamin (1968) explained the effect by suggesting that an association between a CS and US will only form if the organism is surprised by the delivery of the US. Thus, in blocking, A+ trials in Stage 1 of the experiment permit the association of A with the US, which will ensure that the delivery of the US is not surprising on $\mathrm{AX}+$ trials, precluding any conditioning to X. Rescorla and Wagner (1972) agreed with Kamin's analysis and suggested, informally, that "organisms only learn when events violate their expectations" (Rescorla \& Wagner, 1972, p. 75). Importantly, Rescorla and Wagner provided a more formal analysis of the issue by suggesting that conditioning is a function of the arithmetic difference between

This article was published Online First May 20, 2013.

Mark Haselgrove, School of Psychology, The University of Nottingham, United Kingdom; Shu K. E. Tam, Nuffield Laboratory of Ophthalmology, The University of Oxford, United Kingdom; Peter M. Jones, Faculty of Health Sciences, Staffordshire University, United Kingdom.

This research was supported by BBSRC new investigator Grant BB/ F01239X/1 to Mark Haselgrove.

Correspondence concerning this article should be addressed to Mark Haselgrove, School of Psychology, The University of Nottingham, University Park, Nottingham NG7 2RD, United Kingdom. E-mail: Mark.Haselgrove@Nottingham.ac.uk the amount of conditioning supported by the US, and the total associative strength of all CSs present $(\lambda-\Sigma V)$.

Rescorla and Wagner's (1972) formal analysis of conditioning has deservedly enjoyed widespread success both as a consequence of its ability to predict novel behavioral phenomena (e.g., Kremer, 1978) and its applicability to behavioral neuroscience (e.g., Schultz \& Dickinson, 2000; Waelti, Dickinson \& Schultz, 2001). However, it has been known for some time that Rescorla and Wagner's analysis of blocking is incomplete. Dickinson, Hall, and Mackintosh (1976) reported an experiment in which rats first received trials in which presentations of CS A were followed by the immediate delivery of a US, which itself was followed $8 \mathrm{~s}$ later by the delivery of a second US. In Stage 2 of the experiment, one group of rats then went on to receive trials in which an AX compound was paired with the double US sequence just described (i.e., $\mathrm{A}++$ then $\mathrm{AX}++$ ). This group of rats demonstrated a standard blocking effect-showing little evidence of conditioning to $\mathrm{X}$ at test. A second group of rats were treated similarly, but for these animals, in Stage 2, the second US in the double US sequence was omitted (i.e., $\mathrm{A}++$ then $\mathrm{AX}+$ ). This group of rats showed superior conditioned responding to $X$ relative to the first group. That is to say, the omission of the second US enhanced conditioning to the added CS X during Stage 2. According to Rescorla and Wagner (1972, pp. 78-79) the effect of conditioning with either two or one USs is to have different asymptotic levels of conditioning that can be supported by the US $(\lambda)$. It therefore follows that $\mathrm{A}++$ training will result in the associative strength $(\Sigma \mathrm{V})$ of $\mathrm{AX}$ being higher than that which can be supported by the US on $\mathrm{AX}+$ trials, and the expression $(\lambda-\Sigma \mathrm{V})$ will be negative. Consequently, conditioned responding to $\mathrm{X}$ should have been weaker in Dickinson et al.'s second group than in their first group, not stronger.

Although the unblocking effect described by Dickinson et al. (1976) is not as prevalent in the conditioning literature as blocking 
itself, it is still reproducible in a variety of species and in a variety of conditioning procedures. Dickinson et al. employed a clicker and a light as $\mathrm{A}$ and $\mathrm{X}$ in their study of unblocking with rats, using mild electric foot shocks as USs, and the suppression of ongoing lever pressing as the CR. Dickinson and Mackintosh (1979) replicated their basic effect and also showed that unblocking was present in an instrumental conditioning task in which lever pressing resulted in the delivery of one (or two) food pellet USs at the termination of the CSs. Most interestingly, however, this study demonstrated that unblocking was only present when the first and second USs were of the same kind (e.g., shock and shock, or food and food); if the first and second USs were different (e.g., shock and food, or food and shock) then omitting the second US did not result in unblocking. Using a lick suppression procedure with tones and lights as CSs in rats, Mackintosh, Dickinson, and Cotton (1980) demonstrated an unblocking effect when two conditioning trials with $\mathrm{AX}$ were given in Stage 2, but no unblocking was observed when only a single AX trial was given in Stage 2 (see also Mackintosh, Bygrave, \& Picton, 1977).

Rescorla and Colwill (1983) also used lights and tones as CSs and foot shocks as the USs in a conditioned lever-press suppression procedure with rats, and once again demonstrated unblocking by omitting the second US on trials with AX. For these experiments, rats in unblocking groups received training of the form $\mathrm{A}++, \mathrm{AX}+$, while control group rats received $\mathrm{A}+, \mathrm{AX}+$ training. Rescorla and Colwill suggested that the superior conditioned response demonstrated by the unblocking group could have been a consequence of the establishment of an association between $\mathrm{X}$ and A during Stage 2 trials with $\mathrm{AX}+$. Thus, at test, for the unblocking group, an associative chain terminating in two USs may be activated (i.e., $\mathrm{X} \rightarrow \mathrm{A} \rightarrow++$ ) resulting in a strong conditioned response to $\mathrm{X}$. For the blocking group, however, the same association between $\mathrm{X}$ and $\mathrm{A}$ would result in an associative chain that terminates in only one US (i.e., $\mathrm{X} \rightarrow \mathrm{A} \rightarrow+$ ) and a weaker conditioned response to X. In support of this analysis, Rescorla and Colwill demonstrated that presentations of $\mathrm{A}$ in the absence of $\mathrm{X}$ following the $\mathrm{AX}+$ trials in Stage 2 disrupted unblocking, presumably by either undermining the $\mathrm{X}-\mathrm{A}$ association, or the association between A and the US(s). Rescorla and Colwill acknowledged, however, that this analysis of unblocking was less suited to demonstrations of unblocking that employ equivalent Stage 1 training between blocking and unblocking groups (i.e., $\mathrm{A}++$ then $\mathrm{AX}++$ vs. $\mathrm{A}++$ then $\mathrm{AX}+$ ). In order to account for demonstrations of unblocking under these circumstances additional assumptions must be made.

Experiments conducted in Holland's laboratory have demonstrated unblocking by US omission in a number of studies, all of which employed appetitive Pavlovian conditioning with rats. Holland (1984) used visual and auditory CSs as A and X, respectively, and food pellets as the USs. Using video-taped recordings of a variety of conditioned responses, Holland demonstrated unblocking by US omission using a number of behavioral indices (e.g., head jerking, magazine activity). Furthermore, in a comprehensive experiment involving 31 experimental groups, Holland (1984) could find no evidence for the role of Stimulus-Response associations in unblocking by demonstrating that unblocking was abolished in rats that were tested with $\mathrm{X}$ under conditions of satiety. It is notable to add that this experiment failed to replicate Rescorla and Colwill's (1983) abolition of unblocking by presentations of A following AX trials. This manipulation had no additional effect over and above mere context exposure. Holland (1985) was able to show, again using an appetitive Pavlovian conditioning procedure, that conditioned responding was substantially weakened if $\mathrm{AX}++$ training was given in-between the $\mathrm{A}++$ and $\mathrm{AX}+$ stages, a result which he suggested provided support to attentional theories of learning (e.g., Mackintosh, 1975; Pearce \& Hall, 1980). Further studies by Holland (1988) manipulated whether the first and second USs in the double US sequence were the same or different (e.g., food or sucrose), and employed a combination of selective satiety, and retardation and summation tests of a variety of response topographies. These studies suggested that US omission on $\mathrm{AX}+$ trials following $\mathrm{A}++$ trials results both in an enhancement of the association between $\mathrm{X}$ and the first US in the double US sequence, as well as the establishment of inhibition with the second US in the sequence. Bucci, Holland, and Gallagher (1998) demonstrated that a depletion of the cholinergic innervations into the rat homolog of the posterior parietal cortex abolished unblocking in appetitive conditioning. And, finally, using appetitive conditioning in rats, Holland and Kenmuir (2005) demonstrated that amygdala central nucleus lesions abolished unblocking (see also Holland \& Gallagher, 1993), but had no detectable effect on the subsequent superior ability of an unblocked CS to serve as the feature in feature negative discrimination relative to a blocked CS. This final result suggests a dissociation between unblocking, and variations in the associability of the CS.

Unblocking is not only restricted to circumstances in which the number of USs is reduced between trials with A and AX, it is also apparent when the schedule of reinforcement is thinned from a $100 \%$ reinforcement schedule on trials with $\mathrm{A}$, to a $25 \%$ reinforcement schedule on trials with AX. Under these circumstances, Feldman (1971) was able to show that responding to $\mathrm{X}$ was enhanced relative to groups of rats for whom the schedule of reinforcement remained constant between experimental stages either at $25 \%$ and $25 \%$, or at $100 \%$ and $100 \%$. This effect was replicated by Neely and Wagner (1974), but only under circumstances in which trials with $\mathrm{A}$ and $\mathrm{AX}$ were given in discrete stages. If trials with $\mathrm{A}$ and $\mathrm{AX}$ were intermixed, unblocking was abolished. This latter result led Neely and Wagner to suggest the involvement of contextual conditioning in unblocking - an issue to which we will return shortly. Unblocking is also not restricted to studies with rats. Khallad and Moore (1996) used a single subject design with pigeons, a diffuse houselight and a image of a triangle projected onto a keylight as CSs A and X, respectively, and $3 \mathrm{~s}$ access to mixed grain as each US. Their data showed that autoshaped key pecking to $\mathrm{X}$ was superior following $\mathrm{A}++\mathrm{AX}+$ trials than following $\mathrm{A}+\mathrm{AX}+$ trials. Finally, Le Pelley, Oakeshott, and McLaren (2005) were able to obtain an unblocking effect using a causal learning scenario with human participants, but only under circumstances in which the contribution of any inhibitory learning between $\mathrm{X}$ and the second, omitted, US was minimized.

Not all experiments that have employed an $\mathrm{A}++\mathrm{AX}+$ procedure have resulted in unblocking however. Ross (1985) used a conditioned analgesia procedure with rats and found that $\mathrm{A}++$ $\mathrm{AX}+$ training did not enhance responding to $\mathrm{X}$ relative to rats in blocking groups who received either $\mathrm{A}+\mathrm{AX}+$ or $\mathrm{A}++\mathrm{AX}++$ trials. Indeed, if anything, responding in the unblocking group was numerically (but not significantly) weaker than in the blocking 
groups. This result is more in keeping with the predictions of the Rescorla-Wagner model, and is also consistent with the results of experiments in which the intensity or concentration of the US (rather than its number) was reduced between conditioning trials with A and AX. Wagner, Mazur, Donegan, and Pfautz (1980), Cotton, Goodall, and Mackintosh (1982), and Mackintosh and Cotton (1985) all showed that, under these circumstances, X acquired conditioned inhibition.

Although the Rescorla-Wagner model, in its simplest form, struggles to account for unblocking, associative theories that emphasize the role of variations in the amount of attention paid to the CS are more successful (e.g., Mackintosh, 1975; Pearce \& Hall, 1980). We shall return, in greater detail, to these theories in the General Discussion; it is sufficient for the time being to note that both share the assumption that blocking occurs because subjects learn to ignore a CS that is followed by no change in the US. Thus, in a standard blocking experiment, in which the US remains the same between $\mathrm{A}$ and $\mathrm{AX}$ trials, attention to $\mathrm{X}$ will decline across the $\mathrm{AX}+$ trials, hindering the extent to which the animal can associate $\mathrm{X}$ with the US, and thus evoke a conditioned response. Unblocking by US omission follows from these theories because the surprising post-trial event on AX trials constitutes a change in the US, permitting attention to $\mathrm{X}$ to be maintained, and more successful conditioning to $\mathrm{X}$. It also follows from these trials that subjects in the blocking and unblocking groups must learn to ignore or attend to $\mathrm{X}$, respectively. Therefore, unblocking would not be observed after only one trial with AX (Mackintosh et al., 1977; Mackintosh, Dickinson, \& Cotton, 1980).

A second analysis of unblocking emphasizes the role of the experimental context, and by doing so attempts to provide an explanation for unblocking in terms of the Rescorla-Wagner model. Neely and Wagner (1974) noted that the aftereffects of the US may persist between experimental trials, influencing the manner in which the CSs are encoded. For example, following an $\mathrm{A}++$ trial, the effect of the double US sequence might persist until the next trial with $\mathrm{A}++$, influencing the manner in which $\mathrm{A}$ is encoded. In Stage 2, however, the effects of the double US will not be present and A will be encoded in a different manner. Under these circumstances a generalization decrement would be expected on $\mathrm{AX}+$ trials which, if greater than the reduction in $\lambda$ between the double and single USs, would permit $\mathrm{X}$ to gain associative strength on $\mathrm{AX}+$ trials (for a related account see Kremer, Spect, \& Allen, 1980). As Holland (1984) and Dickinson (1980) have noted, not all demonstrations of unblocking by US omission are so amenable to this analysis. For example, Mackintosh, Bygrave, and Picton (1977) gave their rats only a single conditioning trial per day and still demonstrated a reliable unblocking effect. It is unlikely (but not impossible) that the after effects of the US(s) persisted across such long intertrial intervals; and so, a generalization decrement account of the unblocking effect observed by Mackintosh et al. seems equally unlikely. An alternative strategy is to employ an experimental design in which generalization decrement is equated across experimental groups by including an additional CS (B) during Stage 1, that is paired with the complimentary US, or sequence of USs to A (thus, $\mathrm{A}+, \mathrm{B}++$ or $\mathrm{A}++, \mathrm{B}+$ ). Such a design has been implemented, to the best of our knowledge, only once (Holland, 1984).

The purpose of the experiments reported here is twofold. First, we aim to provide demonstrations of unblocking by US omission, in our laboratory, under circumstances in which the contribution of the generalization decrement mechanism suggested by Neely and Wagner (1974) is removed. As noted above, we are aware of only one experiment (Holland, 1984, Experiment 1) in which this factor has been directly balanced across groups. To document the reliability of unblocking under these circumstances is, therefore, of considerable theoretical significance. Second, we aim to investigate whether unblocking can be enhanced. The analysis of unblocking provided by attentional theories of conditioning and learning (e.g., Mackintosh, 1975; Pearce \& Hall, 1980) suggests that the post-trial surprise brought about by the omission of the US on trials with AX maintains attention to X. However, the same theories also predict that US omission should be a suboptimal way of generating unblocking. This follows because the omission of the second US in the double US sequence will, ultimately, become predictable (i.e., not surprising) which should result in a loss of attention to $\mathrm{X}$ and a restriction in any further conditioning. We, therefore, explored in Experiment 2, one way in which to sustain the post-trial surprise throughout Stage 2 trials with $\mathrm{AX}$ in order to see whether unblocking is enhanced.

\section{Experiment 1}

The purpose of Experiment 1 was to demonstrate, in our laboratory, the presence of unblocking by US omission using appetitive Pavlovian conditioning with rats. In particular, we were keen to demonstrate the effect under circumstances in which the contribution of differences in generalization decrement were equalized between experimental groups. The general design of Experiment 1 can be seen in Table 1. During Stage 1, all rats were treated identically; thus, both the Blocking and the Down group received trials in which CS A was paired with the US, which was followed $5 \mathrm{~s}$ later by the delivery of a second US $(\mathrm{A}++)$ and trials in which CS B was paired with only one US $(B+)$. During Stage 2, for the Blocking group, a compound of CSs A and X was paired with the double US sequence $(\mathrm{AX}++)$ and $\mathrm{CS} B$ was paired with only one US $(B+)$. During Stage 2 for the Down group, however, the AX compound was now followed by one US $(\mathrm{AX}+)$ and $\mathrm{B}$ was followed by the double US sequence $(\mathrm{B}++)$. In a final test session, nonreinforced trials with $\mathrm{X}$ presented in isolation were given. On the basis of the experiments described in our review of the literature, the surprising omission of the second US on the $\mathrm{AX}+$ trials in the Down group should result in stronger conditioned responding to $\mathrm{X}$, relative to the Blocking group. Importantly, however, both groups experience an equal number of trials with both a single US, and a double US sequence during Stage 1 and Stage 2 of the experiment. Consequently, the contribution of

Table 1

The Design of Experiment 1

\begin{tabular}{lccc}
\hline Group & Stage 1 & Stage 2 & Test \\
\hline Blocking & $\mathrm{A}++\mathrm{B}+$ & $\mathrm{AX}++\mathrm{B}+$ & $\mathrm{X}$ \\
Down & $\mathrm{A}++\mathrm{B}+$ & $\mathrm{AX}+\mathrm{B}++$ & $\mathrm{X}$ \\
\hline
\end{tabular}

Note. A, B, and $\mathrm{X}$ refer to experimental CSs, $15 \mathrm{~s}$ in duration. + is the delivery of a single food pellet immediately upon the termination of the CS. ++ is the delivery of a single food pellet immediately upon the termination of the CS, followed $5 \mathrm{~s}$ later by the delivery of a pair of food pellets. 
generalization decrement that is engendered by the difference between ++ and + trials is balanced between the groups, and the analysis of unblocking provided by Neely and Wagner (1974) would not apply here.

\section{Method}

Subjects. Sixteen male Lister hooded rats (Rattus norvegicus) served as subjects. These were supplied by Harlan Olac (Bicester, Oxon, United Kingdom) and housed in pairs in a light-proof holding room that was illuminated for $12 \mathrm{hr} /$ day. Rats received all experimental sessions during the light portion of this cycle. Rats were fed a restricted diet in order to reduce them to not less than $85 \%$ of their initial free-feeding weights, and their weights were subsequently allowed to increase in proportion to a separate group of rats that were kept in the same room and allowed free access to food. Before the beginning of the experiment, eight rats were assigned to each of the Blocking and Down groups. Immediately prior to the first experimental session, the mean weight of rats was $220 \mathrm{~g}$ for the Down group and $241 \mathrm{~g}$ for the Blocking group.

Apparatus. Eight identically specified conditioning chambers (MED Associates, St. Albans, VT) were used, each measuring 30.0 $\mathrm{cm} \times 24.0 \mathrm{~cm} \times 20.5 \mathrm{~cm}$ high. The ceiling and longer walls, one of which served as a door, were made from clear acrylic. The shorter walls were made from metal plates, and the floor was constructed of a series of 4.8-mm diameter stainless steel rods, running parallel to the shorter walls and spaced $1.6 \mathrm{~cm}$ apart, center to center. One of the shorter walls was fitted with a squareshaped recessed magazine of $5 \mathrm{~cm}$ diameter, to which $45-\mathrm{mg}$ food pellets (standard formula; Noyes, Lancaster, NH) could be delivered. Food pellets could be delivered following presentations of stimuli-a single pellet delivered at the offset of a CS is hereafter referred to as US1, and a sequence of two food pellets delivered $5 \mathrm{~s}$ after the offset of a CS is referred to as US2. Both US1 and US2 could be delivered on the same trial. This procedure, in which the delivery of a single food pellet serves as US1 and the delivery of a pair of food pellets serves as US2, replicates the procedure employed in other successful demonstrations of unblocking in appetitive Pavlovian conditioning (e.g., Holland, 1984; Holland \& Kenmuir, 2005). An infrared beam was sent from one lateral side of the magazine to the other, and each interruption of this beam could be recorded as magazine activity. The magazine was located equidistant from the two longer walls, and $1.8 \mathrm{~cm}$ above the grid floor at its lowest edge. A pair of 2.8-W incandescent lamps whose 2.5-cm diameter, circular covers were composed of opaque plastic were located symmetrically to either side of the food tray. Each was $2.5 \mathrm{~cm}$ in diameter, and located $10.5 \mathrm{~cm}$ from the grid floor. The distance between the lamps was $16.0 \mathrm{~cm}$, center to center. These lamps could be alternately illuminated, such that each was lit for $0.1 \mathrm{~s}$ at a time, and the onset of illumination for one lamp was contiguous with the offset of illumination for the other. This was used as an experimental stimulus, hereafter referred to as the alternating light. A third 2.8-W lamp, shielded from directly illuminating the floor by a metal hood, was positioned on the opposite wall, $17.5 \mathrm{~cm}$ above the floor and equidistant from the two longer walls. Constant illumination of this lamp was used as a second visual stimulus, and is referred to as the rear light. The wall containing the rear light was also equipped with a loudspeaker, which could produce a $1.5-\mathrm{kHz}, 92-\mathrm{dB}$ pure tone. Operation of the alternating light, the rear light, and the tone, as well as the delivery of US1 and US2, was controlled by a computer that was programmed with the MED-PC programming language. Each of the three CSs was $15 \mathrm{~s}$ in duration. Each conditioning chamber was individually housed in a sound- and light-attenuating box, which was fitted with an exhaust fan that provided ventilation and a constant background noise of $68 \mathrm{~dB}$.

Procedure. Rats were initially trained to retrieve food from the magazine in a single session. This session was $30 \mathrm{~min}$ in duration, and a single food pellet was delivered to the magazine every $60 \mathrm{~s}$. All rats successfully consumed all pellets during this session. Following this, Stage 1 training consisted of 10 sessions with $\mathrm{A}++$ and $\mathrm{B}+$. Each presentation of $\mathrm{A}$ was followed immediately by US1, and $5 \mathrm{~s}$ later by US2; each presentation of B was followed by US1 only. Sessions during this stage were $52 \mathrm{~min}$ in duration, and contained 12 trials with each of $\mathrm{A}$ and $\mathrm{B}$, in a random sequence. The intertrial interval (ITI), defined as the length of time between the end of one CS presentation and the beginning of the next, varied around a mean of $105 \mathrm{~s}$ and had a range of 65-145 s. For half the animals, the rear light served as A and the alternating panel lights served as B; for the remaining half, this was reversed. This counterbalancing was orthogonal to the assignment of rats to the Down and Blocking groups. The use of eight conditioning chambers enabled eight rats to be trained simultaneously, with each cohort of rats consisting of one experimental group. The order in which these cohorts were trained was varied between sessions, so that no systematic difference between the times at which groups received training was present.

Five sessions of training were given in Stage 2. The Down group received $\mathrm{AX}+, \mathrm{B}++$ training; the Blocking group received $\mathrm{AX}++, \mathrm{B}+$ training. Each session contained 12 trials of each type, in a random sequence. The tone served as $\mathrm{X}$ for all animals, and was presented in simultaneous compound with A. Other details of these sessions were the same as for Stage 1. Finally, rats received a test session that was intended to assess conditioned responding to $\mathrm{X}$. This session contained both training trials and test trials. The first 10 trials of the session consisted of five trials with each of AX and B, with the same pattern of US1 and US2 delivery used in Stage 2, in a random sequence. The next two trials were presentations of $\mathrm{X}$ alone, which were not followed by the delivery of any food. Rats then received five more training trials with each of AX and B in the same manner as earlier trials, followed by two final presentations of $X$. This test session, therefore, contained four test trials with $\mathrm{X}$. All other details of this test session were the same as for Stages 1 and 2.

\section{Results and Discussion}

A Type I error rate of $p<.05$ was adopted for all of the statistical tests in this and the subsequent experiment.

Stage I. The Blocking and Down groups were treated identically during Stage 1 of the experiment, therefore, the mean number of responses per minute during A and B for each of the 10 sessions of Stage 1 were collapsed across the two groups and are shown in Figure 1. Conditioning proceeded smoothly across the 10 sessions, with responding higher to $\mathrm{A}$ than to $\mathrm{B}$. This impression was confirmed with a two-way analysis of variance (ANOVA) of individual mean responses per minute with the variables of session (1 to 10) and CS (A and B) which revealed an effect of session, 


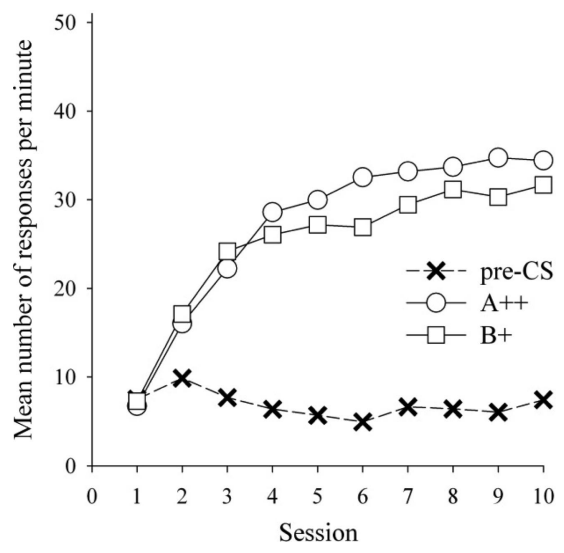

Figure 1. The mean number of responses per minute during A, B, and the pre-CS period across the 10 sessions of Stage 1 of Experiment 1. Results are collapsed across the two Experiment groups.

$F(9,135)=33.70, M S E=67.65$, an effect of CS, $F(1,15)=$ 18.00, $M S E=19.33$, and an interaction between these variables, $F(9,135)=2.85, M S E=17.23$. Simple effects analysis of this interaction revealed a difference between A and B on Sessions 6, 7 and $9, F \mathrm{~s}(1,150)>6.45, M S E=17.44$, but not on the remaining session, $F \mathrm{~s}(1,150)<3.63, M S E=17.44$.

Stage II. The mean number of responses per minute during $\mathrm{AX}$ and $\mathrm{B}$ in the Down and Blocking groups across the five sessions of Stage 2 are shown in the top and bottom panels, respectively, of Figure 2. Responding to AX and B increased marginally in both groups during this stage, however, there was little indication of any difference between the two groups in responding to either AX or B. A three-way ANOVA of individual mean rates of responding with the variables of group (Blocking, Down), CS (AX and B) and session (1 to 5) revealed an effect of session, $F(4,56)=5.82, M S E=48.58$, but no effect of group, of $\mathrm{CS}$, and no interaction between these variables, $F \mathrm{~s}(1,14)<1.11$, $M S E s>87.04$, and no interactions between these variables and the variable of session, $F \mathrm{~s}(4,56)<2.40, M S E s>23.56$. Also shown in the panels of Figure 2 are the mean number of responses per minute made during the 15-s intervals prior to all the CSs in the two groups. A two-way ANOVA of individual mean responses per minute with the variables of group (Blocking, Down) and session (1-15) revealed no effect of group, no effect of session, and no interaction between these variables, all $F \mathrm{~s}<1$.

Test. The mean number of responses per minute made during the test session by the two groups during the four presentations of $\mathrm{X}$ is shown, in two trial blocks, in Figure 3. Overall, responding during $\mathrm{X}$ was greater in group Down than in group Blocking; however, this effect was diminished by the second block of two trials, presumably because of the nonreinforced trials with $\mathrm{X}$. These impressions were confirmed with a two-way ANOVA of individual responses per minute with the factors of group (Blocking, Down) and trial block (1 and 2) which revealed no effect of trial block, $F(1,14)=3.17, M S E=69.64$, but an effect of group, $F(1,14)=16.25, M S E=155.07$, and a group $\times$ trial block interaction, $F(1,14)=13.28, M S E=69.64$. Simple effects analysis revealed that the groups differed on trial block 1 , $F(1,28)=28.92, M S E=112.36$, but not on trial block 2 ,
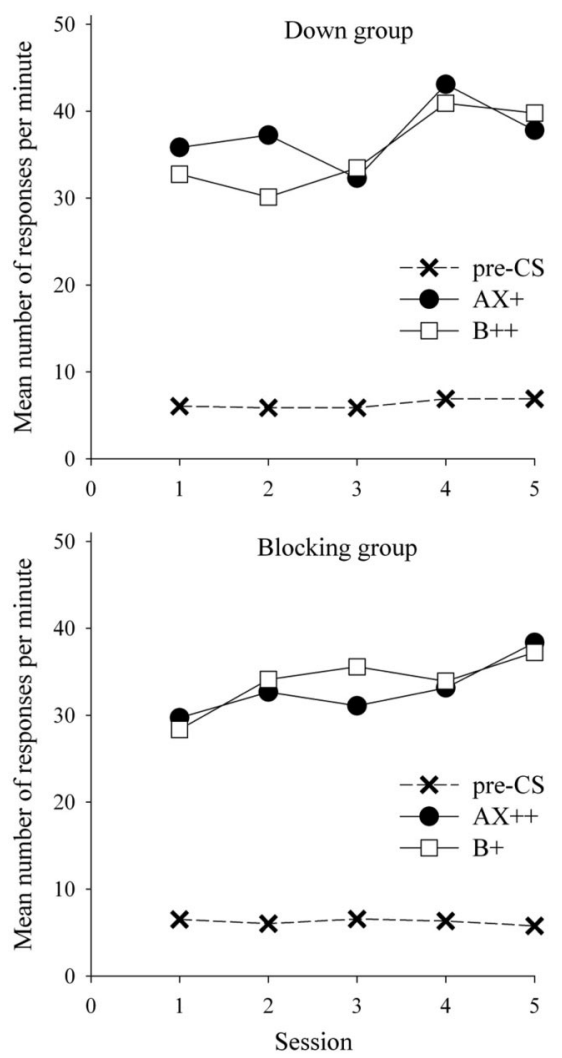

Figure 2. The mean number of responses per minute during AX, B, and the pre-CS period across the five sessions of Stage 2 of Experiment 1. Top panel: Results from the Down group. Bottom panel: Results from the Blocking group.

$F(1,28)=1.74, M S E=112.36$. An identical ANOVA was performed upon the individual number of responses per minute made during the 15-s intervals prior to the test trials with $\mathrm{X}$. This revealed an effect of trial block, $F(1,14)=7.99, M S E=31.70$, but no effect of group, and no group $\times$ trial block interaction, $F \mathrm{~s}<1$.

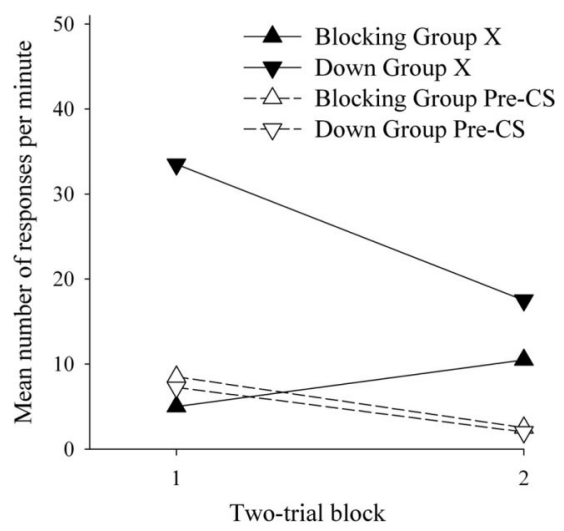

Figure 3. The mean number of responses per minute during $\mathrm{X}$ and the pre-CS period for the Blocking and Down groups across the 2 two-trial blocks of the test session of Experiment 1. 
These results constitute a successful demonstration of unblocking by US omission. To the best of our knowledge, this effect has been reported in appetitive conditioning in rats in only one other laboratory (e.g., Holland, 1984, 1985, 1988; Holland \& Gallagher, 1993; Holland \& Kenmuir, 2005). The experimental design employed in Experiment 1 deliberately matched the Blocking and Down groups on the number of trials in which a single US and a double US sequence were presented during the two conditioning stages. The generalization decrement mechanism proposed by Neely and Wagner (1974) therefore provides an inappropriate analysis of Experiment 1. Furthermore, Experiment 1 is not amenable to the kind of within-compound association analysis provided by Rescorla and Colwill (1983). Rats in both the Blocking and the Down groups received trials in Stage 1 with $\mathrm{A}++$. Therefore, the associative chain from $\mathrm{X}$ would, for both groups, terminate in two USs (i.e., $\mathrm{X} \rightarrow \mathrm{A} \rightarrow++$ ). On this basis alone, therefore, conditioned responding to $\mathrm{X}$ would be equivalent in the two groups. We shall consider in the General Discussion how elaborations of the analyses provided by Neely and Wagner, and by Rescorla and Colwill might be able to address some of these issues. For the time being, however, it is sufficient to note that these analyses, without further assumptions granted, provide inadequate explanations for the results of Experiment 1. It is further worth noting that Experiment 1 constitutes a demonstration of stronger conditioned responding in the group of animals that were given paired presentations of $\mathrm{X}$ with one US, than a group of rats given paired presentations of $\mathrm{X}$ with two USs. This is quite a counter intuitive result and one that seems, at face value, rather difficult to explain by analyses of blocking that emphasize the role of rational inference (e.g., Beckers, Miller, De Houwer, \& Urushihara, 2006).

\section{Experiment 2}

Although the accounts of unblocking provided by Neely and Wagner (1974) and Rescorla and Colwill (1983) are ill suited to the results of Experiment 1, attentional theories of conditioning are more successful. According to the theories proposed by Mackintosh (1975) and Pearce and Hall (1980), the surprising omission of the second US on $\mathrm{AX}+$ trials in the Down group should counter the decrement in attention to $\mathrm{X}$ that animals in the Blocking group will encounter on $\mathrm{AX}++$ trials. Therefore, animals in the Down group will be better able to associate the more salient $X$ with the trial outcome (and consequently evoke a stronger conditioned response) than animals in the Blocking group for whom $\mathrm{X}$ is rather less salient. However, attentional theories of conditioning predict that, eventually, the omission of the second US will become predictable on $\mathrm{AX}+$ trials. Therefore, attention to $\mathrm{X}$ will, ultimately, fall-restricting future conditioning with $X$. The purpose of Experiment 2 was to examine whether unblocking can be enhanced by arranging for more favorable conditions of post-trial surprise, and the general design of the experiment can be seen in Table 2. In Stage 1, three groups of rats all received training that was identical in form to that described in Stage 1 of Experiment 1. Thus, on each trial, all subjects received trials with A that were followed by a US, which itself was followed $5 \mathrm{~s}$ later by a second US $(\mathrm{A}++)$, and trials with B that were followed by only one US $(\mathrm{B}+)$. In Stage 2, a Blocking group and a Down group then received trials that were identical in form to the training received
Table 2

The Design of Experiment 2

\begin{tabular}{lllc}
\hline Group & Stage 1 & \multicolumn{1}{c}{ Stage 2 } & Test \\
\hline Blocking & $\mathrm{A}++\mathrm{B}+$ & $\mathrm{AX}++\mathrm{AX}++\mathrm{B}+\mathrm{B}+$ & $\mathrm{X}$ \\
Down & $\mathrm{A}++\mathrm{B}+$ & $\mathrm{AX}+\mathrm{AX}+\mathrm{B}++\mathrm{B}++$ & $\mathrm{X}$ \\
Partial & $\mathrm{A}++\mathrm{B}+$ & $\mathrm{AX}++\mathrm{AX}+\mathrm{B}++\mathrm{B}+$ & $\mathrm{X}$ \\
\hline
\end{tabular}

Note. A, B, and $\mathrm{X}$ refer to experimental CSs, $15 \mathrm{~s}$ in duration. + is the delivery of a single food pellet immediately upon the termination of the CS. ++ is the delivery of a single food pellet immediately upon the termination of the CS, followed $5 \mathrm{~s}$ later by the delivery of a pair of food pellets.

by their namesakes in Experiment 1. Thus, for the Blocking group, a compound of $\mathrm{A}$ and $\mathrm{X}$ was paired with the double US sequence $(\mathrm{AX}++)$ and CS B was paired with only one US $(\mathrm{B}+)$; and for the Down group, the AX compound was followed by one US $(\mathrm{AX}+)$ and $\mathrm{B}$ was followed by the double US sequence $(\mathrm{B}++)$. Animals in a Partial group received trials that were intended to maintain the post-trial outcome as entirely unpredictable. Thus, for this group, half of the trials with $\mathrm{AX}$ terminated in just one US, and the remaining trials with $\mathrm{AX}$ terminated in a US which itself was followed $5 \mathrm{~s}$ later by a second US. The same treatment was also applied to B in this group. According to the Pearce-Hall model, for example, ensuring that the post-trial outcome is never predictable should sustain a high level of attention to X indefinitely, increasing the opportunity that this stimulus has to associate with the US. This is in contrast to the unblocking group, who will enjoy the benefits of the omission of the second US for only as long as it remains surprising. We can, therefore, derive two predictions for Experiment 2. First, to observe a replication of the unblocking effect demonstrated in Experiment 1 - conditioned responding to $\mathrm{X}$ should be stronger in the Down group than in the Blocking group. And second, to see an enhancement of unblocking-superior conditioned responding to $\mathrm{X}$ in the Partial group than in the Down group.

\section{Method}

Subjects and apparatus. Twenty-four male rats served as subjects, from the same stock and housed in the same conditions as for Experiment 1. Rats were fed a restricted quantity of food in the same way as for Experiment 1, and were assigned to the Blocking, Down, and Partial groups. The mean weights of rats in these groups at the start of the experiment were 269, 270, and $268 \mathrm{~g}$, respectively. The apparatus used was the same as for Experiment 1.

Procedure. The overall design of Experiment 2 is shown in Table 2. Rats were trained to retrieve food from the magazine in the same manner as for Experiment 1. Ten sessions of $\mathrm{A}++, \mathrm{B}+$ training were then given, in the same way as for Experiment 1. The sequence in which groups were trained during this and subsequent stages of the experiment was varied from session to session. During Stage 2, the Blocking and Down groups received the same treatments as for Experiment 1. The Partial group received five sessions of $\mathrm{AX}+/++, \mathrm{B}+/++$ training, during which each of $\mathrm{AX}$ and B was followed by US1 on every trial and US2 on $50 \%$ of trials. The presence or absence of US2 was random, with the constraint that it was delivered following six of each trial type during each session. For the first test session, the sequence of 
training and test trials was identical to Experiment 1, and all rats received training trials of the type given during Stage 2. For the Partial group, the delivery of US2 on training trials was random, with the constraint that each of AX and B was followed by US2 on five of its 10 trials. Rats then received an additional five sessions of training in the same way as for Stage 2, followed by a second test session that was identical to the first. All details that have been omitted here were the same as for Experiment 1.

\section{Results and Discussion}

Stage I. The Partial, Blocking, and Down groups were treated identically during Stage 1 of the experiment, therefore, the mean number of responses per minute during $\mathrm{A}$ and $\mathrm{B}$ on each of the 10 sessions of Stage 1 were collapsed across the three groups and are shown in Figure 4. Conditioning proceeded smoothly across the 10 sessions, with responding higher to A than to B. This impression was confirmed with a two-way ANOVA of individual mean responses per minute with the variables of session (1 to 10) and CS (A and B) which revealed an effect of session, $F(9,207)=24.72$, $M S E=96.31$, an effect of CS, $F(1,23)=77.64, M S E=24.01$, and an interaction between these factors that fell short of statistical significance, $F(9,207)=1.82, M S E=18.24, p=.067$ (simple effects analysis of this interaction revealed a difference between A and $\mathrm{B}$ on Sessions 2 to $10, F \mathrm{~s}(1,230)>4.58, M S E=18.82$, but not on Session $1, F<1$ ).

Stage II. The mean number of responses per minute during $\mathrm{AX}$ and $\mathrm{B}$ in each of the three groups across the 10 sessions of Stage 2 are shown in the top, center, and bottom panels of Figure 5. During the first five sessions of Stage 2, conditioned responding was relatively stable for the three groups, although there was a tendency, in the Partial group, of an increase and then a decrease in responding. During the second group of five sessions of Stage 2 , responding was more variable. Responding increased in the Down group, and fell in the Partial Group. A three-way ANOVA of individual mean rates of responding with the variables of group (Blocking, Down, and Partial), CS (AX and B), and session (1 to 10) revealed no effects of session, $F(9,189)=1.60, M S E=$ 123.88 , and no effect of CS, $F<1$. There was, however, an effect of group, $F(2,21)=5.73, M S E=568.41$. The group $\times \mathrm{CS}$

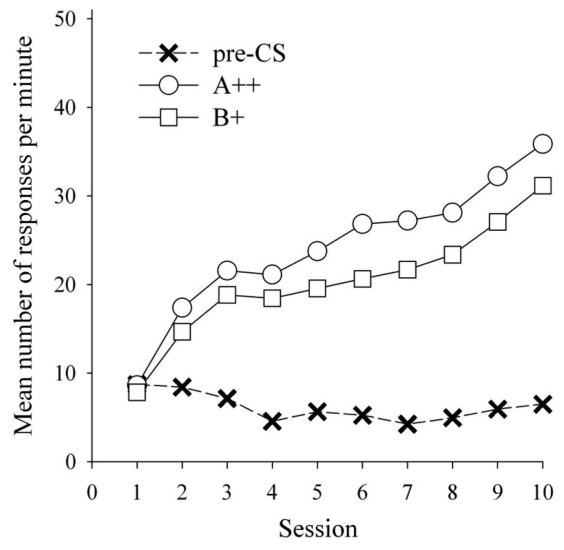

Figure 4. The mean number of responses per minute during A, B, and the pre-CS period across the 10 sessions of Stage I of Experiment 2. Results are collapsed across the three Experiment groups.
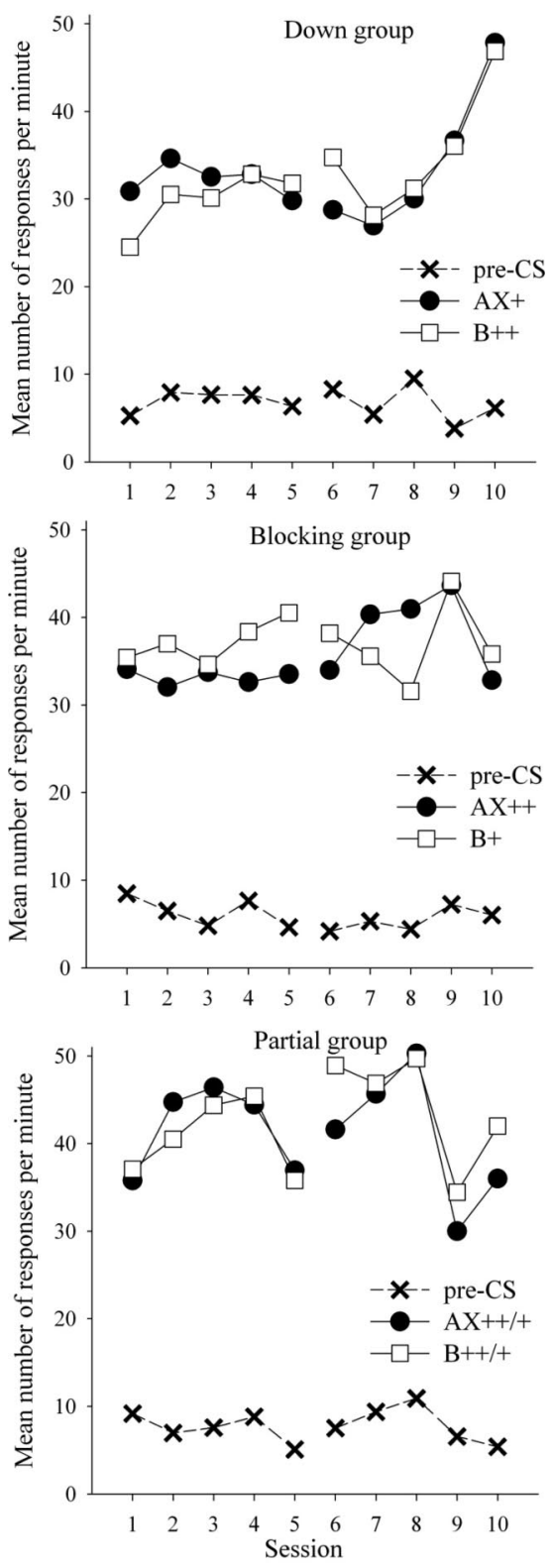

Figure 5. The mean number of responses per minute during AX, B, and the pre-CS period across the 10 sessions of Stage 2 of Experiment 2. Top panel: Results from the Down group. Centre panel: Results from the Blocking group. Bottom Panel: Results from the Partial group.

interaction, $F<1$, the session $\times \mathrm{CS}$ interaction, $F(9,189)=1.74$, $M S E=48.36$, and the three-way interaction, $F(18,189)=1.22$ $M S E=48.36$, were all nonsignificant. There was, however, a significant group $\times$ session interaction, $F(18,189)=3.82, M S E=$ 123.88. Simple effects analysis of this interaction revealed that, collapsed across the two CSs, the three groups differed on Sessions 3 and 4 , and 6 to $10, F \mathrm{~S}(2,210)>3.33, M S E=168.33$. Bonferroni/Dunn posthoc tests (critical differences between 10.47 and 15.00) revealed that responding in the Partial group was significantly higher than the down group on Sessions 3, 6, 7, and 8. In addition, on Session 8, responding in the Partial group was 
significantly higher than in the Blocking group. The differences among the means highlighted by the overall ANOVA on Sessions 4, 9, and 10 did not survive the more stringent criteria of the post hoc tests. Also shown in the panels of Figure 5 are the mean number of responses per minute made during the 15-s intervals prior to the CSs in the three groups. A two-way ANOVA of individual mean responses per minute with the variables of group (Blocking, Down, and Partial) and session (1-10) revealed no effect of group, $F(2,21)=1.16, M S E=56.66$, no effect of session, $F(9,189)=1.43, M S E=15.66$, and no interaction between these factors, $F(18,189)=1.46, M S E=15.66$.

Test. The mean number of responses per minute made by the three groups during the four presentations of $\mathrm{X}$ during the two test sessions are shown in the top panel of Figure 6. The first thing to note from this panel is that the two test sessions revealed very similar patterns of data: across both tests, responding was higher in the Partial and Down groups than in the Blocking group- unblocking effects. In addition, however, on the first trial of both tests, responding was higher in the Partial group than in the Down
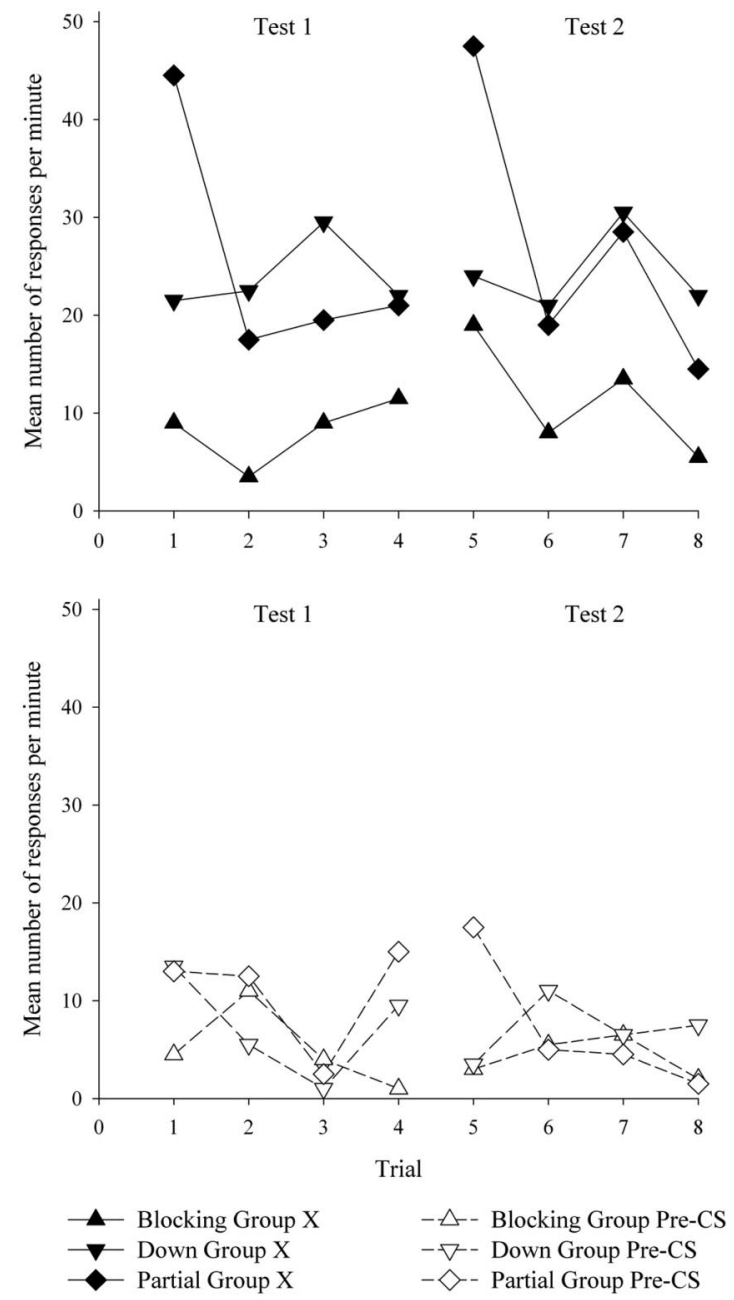

Figure 6. The mean number of responses per minute during $\mathrm{X}$ (top panels) and the pre-CS period (bottom panels) for the Blocking, Down, and Partial groups across the four trials of Tests 1 and 2 of Experiment 2. group - an enhancement of unblocking. These impressions were confirmed with a three-way ANOVA of individual responses per minute with the variables of group (Blocking, Down, and Partial), test (1 and 2), and trial (1 to 4) which revealed an effect of group, $F(2,21)=6.39, M S E=810.19$, and of trial, $F(3,63)=4.17$, $M S E=377.40$ but no effect of test, $F<1$. None of the Group $\times$ Test, Test $\times$ Trial, or Group $\times$ Test $\times$ Trial interactions were significant, $F \mathrm{~s}<1$, However, there was a significant Group $\times$ Trial interaction, $F(6,63)=2.26, M S E=377.40$. Simple effects analysis of this interaction revealed first, an effect of trial for the Partial group, $F(3,63)=7.51, M S E=377.40$, but not the remaining groups, $F \mathrm{~S}<1$; and second, a difference among the groups on Trial $1, F(2,84)=9.01, M S E=485.60$. The difference among the groups on Trial 3 approached significance, $F(2,84)=$ 3.02, $p=.054, M S E=485.60$. Bonferroni/Dunn post hoc tests (critical difference $=22.49$ ) revealed responding on the average of the first trial of each test session was higher in the Partial group than either the Down, or the Blocking groups. Returning to the overall ANOVA, Bonferroni/Dunn post hoc tests of the significant main effect of group revealed that, across the two test sessions as a whole, the Blocking group was significantly lower than both the Down group and the Partial group, critical difference $=14.9$. An identical ANOVA was performed upon the individual number of responses per minute made during the 15 -s intervals prior to the test trials with $X$ (bottom panel of Figure 6). This revealed no effect of trial, $F(3,63)=1.20$, no effect of group or session, $F \mathrm{~S}<1$ and no interactions among any of the variables, $F \mathrm{~s}<1$.

The results obtained from the Down and Blocking groups replicated those obtained in Experiment 1. Thus, our observation of unblocking by US omission is reliable. More importantly, however, conditioned responding to $\mathrm{X}$ in the Partial group was significantly stronger than in either the Blocking or Down groups, confirming that the treatment received by the Partial group did indeed enhance unblocking. It is notable that this effect was only present on the first trial of each of the two test sessions. After this trial, responding to $\mathrm{X}$ in the Partial group declined to levels that were comparable to the Down group. It remains to be determined why the enhancement of unblocking in the Partial group should be so ephemeral. One possibility is that attention to $\mathrm{X}$ was so high in the Partial group that the nonreinforced test trials resulted in particularly rapid extinction. To circumvent this problem, future study of this effect could consider testing $\mathrm{X}$ with single probe trials presented infrequently, across training in Stage 2, to minimize the effect of extinction.

The superior conditioned responding in the Partial group relative to the Blocking group resembles the results reported by Feldman (1971), and Neely and Wagner (1974). Recall that in these experiments, rats in a Down group first received trials in which A was paired with the US on every trial. In Stage 2 rats in the Down group received trials in which the reinforcement schedule was thinned to $25 \%$. Conditioned responding to $\mathrm{X}$ was superior in this group relative to a Blocking group who had a thin (25\%) reinforcement schedule with A and AX in both stages of the experiment. Where the current experiment is novel, however, is with the demonstration of superior unblocking in the Partial group relative to a group which is itself also demonstrating an unblocking effect (Down group). 


\section{General Discussion}

Two appetitive conditioning experiments with rats examined the conditions under which the omission of an expected US enhanced conditioning to a novel cue-unblocking. We arranged for all experimental groups in Experiments 1 and 2 to experience trials that terminated with both a single US and a double US sequence. In doing so we were able to balance, among the groups, any effects of generalization decrement that might arise from changing the nature of the US on trials with A and AX (Neely \& Wagner, 1974). Furthermore, conditioning trials with A in Stage 1 were followed by two USs for all of the experimental groups in these experiments. If we assume that the strength of the within-compound association between $\mathrm{X}$ and $\mathrm{A}$ was equivalent among the groups in the current experiment (an assumption to which we will soon return) then the contribution of this mechanism to our demonstration of unblocking should similarly be equivalent across the experimental groups (Rescorla \& Colwill, 1983). Most importantly, Experiment 2 demonstrated that presentations of $\mathrm{AX}$ that were followed by either a single US or a double US sequence, in an unpredictable sequence, resulted in an enhancement of unblocking. This result is particularly consistent with theories of blocking and unblocking that emphasize the importance of post-trial surprise in the maintenance of attention to CSs (e.g., Mackintosh, 1975; Pearce \& Hall, 1980).

It is appropriate to describe, in more formal terms, exactly how the Pearce-Hall (1980) and Mackintosh (1975) theories are able to account for the current data. We present these two analyses in turn before turning our attention to other accounts of unblocking. According to Pearce and Hall ${ }^{1}$, the acquisition of associative strength to a $\mathrm{CS}(\Delta \mathrm{V})$ will proceed according to Equation 1:

$$
\Delta \mathrm{V}=\alpha \mathrm{S} \lambda
$$

In which $\mathrm{S}$ is a fixed learning rate parameter, $\lambda$ is the asymptote of conditioning supported by the US and $\alpha$ is the associability of the CS, the value of which, on trial $n\left(\alpha^{\mathrm{n}}\right)$, is shown in Equation 2.

$$
\alpha^{n}=\left|\lambda^{\mathrm{n}-1}-\sum \mathrm{V}^{\mathrm{n}-1}\right|
$$

In which $\lambda^{\mathrm{n}-1}$ is the asymptote of conditioning supported by the US on trial $n-1$ and $\Sigma V^{n-1}$ is the total associative strength of all CSs present on trial n-1. According to Pearce and Hall, $\mathrm{A}++$ trials will result in associations forming between A and the first US, and between A and the second US (henceforth US1 and US2). Equation 2 will, therefore, ensure that the associability $(\alpha)$ of A will decline rapidly across Stage 1 for all of the experimental groups. Equation 2 will also ensure that, after the first $\mathrm{AX}++$ trial in the Blocking groups, $\alpha$ will decline rapidly to $\mathrm{X}$. This follows because both US1 and US2 are fully predicted on the AX trials by A $\left(\mathrm{V}_{\mathrm{AX}-\mathrm{US} 1} \approx \lambda_{1}\right.$ and $\left.\mathrm{V}_{\mathrm{AX} \text {-US2 }} \approx \lambda_{2}\right)$. Consequently the change in the associative strength of $\mathrm{X}$ will be close to zero (Equation 1). However, if US2 is omitted on trials with AX (as was the case for the Down groups) then $\mathrm{AX}$ predicts the presence of something that does not occur $\left(\mathrm{V}_{\mathrm{AX}-\mathrm{US} 2} \neq \lambda_{2}\right)$ and $\alpha_{\mathrm{X}}$ will be restored to a nonzero level, permitting conditioning (Equation 1). However, this restoration will be transient, as eventually the AX compound will become a good signal for the absence of US2 $\left(\bar{\lambda}_{2}\right)$. Once this is complete, attention to $\mathrm{X}$ will again be low $\left(\alpha_{X} \approx 0\right)$ and conditioning will cease. In order to prevent attention to $X$ being driven to zero, the AX trials must never fully predict whether US2 will be present or absent (i.e., $\mathrm{V}_{\mathrm{AX}-\mathrm{US} 2} \neq \lambda_{2}$ and $\overline{\mathrm{V}}_{\mathrm{AX} \text {-US2 }} \neq \bar{\lambda}_{2}$, thus, sustaining attention to $\mathrm{X}$ indefinitely and permitting further conditioning. These are precisely the circumstances that prevailed in Stage 2 of Experiment 2 for the Partial group.

It must be noted, however, that the explanation provided by the Pearce and Hall (1980) model for the standard unblocking effect does encounter difficulties if it is assumed that the AX compound enters Stage 2 with asymptotic associative strength for US1 $\left(\mathrm{V}_{\mathrm{AX} \text {-US1 }}=\lambda_{1}\right)$. Under these circumstances, in Stage 2 , the omission of US2 on AX + trials will result in an increase in $\alpha_{\mathrm{A}}$ and $\alpha_{X}$, permitting the acquisition of excitatory associative strength to $\mathrm{A}$ and $\mathrm{X}$. However, if $\mathrm{V}_{\mathrm{AX}-\mathrm{US} 1}=\lambda_{1}$ on Trial 1 of Stage 2 then the acquisition of additional excitatory associative strength will result in $\mathrm{V}_{\text {AX-US1 }}$ exceeding $\lambda_{1}$. The consequent overexpectation will then be corrected with the acquisition of inhibitory associative strength (or a CS-noUS1 association) which will balance any additional excitation acquired by $\mathrm{X}$; thus, cancelling out any unblocking effect. To circumvent this prediction, one can make the assumption that conditioning does not proceed to asymptote in Stage 1 in the Down and Blocking groups, and/or introducing $\mathrm{X}$ to A in Stage 2 results in generalization decrement, reducing the total associative strength of the AX compound to a subasymptotic level.

According to Mackintosh (1975) the acquisition of associative strength to a target $\mathrm{CS}\left(\Delta \mathrm{V}_{\mathrm{T}}\right)$ will proceed according to Equation 3:

$$
\Delta \mathrm{V}_{\mathrm{T}}=\alpha_{\mathrm{T}} \beta\left(\lambda-\mathrm{V}_{\mathrm{T}}\right)
$$

In which $\alpha_{T}$ is the associability of the target CS, $\beta$ is a learning rate parameter determined by the properties of the US and $\lambda$ is the asymptote of conditioning supported by the US. According to Mackintosh (1975) the associability $(\alpha)$ of a CS increases if it is a better predictor of the US than all other CSs present on a trial, and decreases if it is no better a predictor of the US than all the other CSs present on a trial. The rules specified by Mackintosh for determining these increases and decreases to a target CS (T) are shown in Equations $4 \mathrm{a}$ and $4 \mathrm{~b}$ :

$$
\begin{aligned}
& \Delta \alpha_{\mathrm{T}}>0 \text { if }\left|\lambda-\mathrm{V}_{\mathrm{T}}\right|<\left|\lambda-\mathrm{V}_{\mathrm{r}}\right| \\
& \Delta \alpha_{\mathrm{T}}<0 \text { if }\left|\lambda-\mathrm{V}_{\mathrm{T}}\right| \geq\left|\lambda-\mathrm{V}_{\mathrm{r}}\right|
\end{aligned}
$$

Where $V_{r}$ is the sum of the associative strength of all available CSs, minus $\mathrm{V}_{\mathrm{T}}$ (that is to say, the remainder). The size of the change in $\alpha$ is assumed to be proportional to the magnitude of the inequalities in Equation $4 \mathrm{a}$ and $4 \mathrm{~b}$. According to Mackintosh, $\mathrm{A}++$ trials will result in associations forming between $\mathrm{A}$ and US1, and A and US2. However, unlike the Pearce and Hall (1980) model, because A is a better predictor of these USs than any other available CS (such as the experimental context), Mackintosh's model predicts attention to A will increase during Stage 1. After the first $\mathrm{AX}++$ trial in the Blocking groups, Equation $4 \mathrm{~b}$ will ensure that attention will decline rapidly to $\mathrm{X}$. This follows be-

\footnotetext{
${ }^{1}$ For ease of exposition we describe here the version of the theory provided by Pearce and Hall (1980). Pearce, Kaye, and Hall (1982; see also: Le Pelley, Haselgrove, \& Esber, 2012) expanded upon the theory to avoid a number of regrettable predictions. Again, for ease of exposition, we also avoid a detailed discussion of the mechanisms involved in inhibitory learning, but, as will be seen, acknowledge their role in the overexpectation that will take place on $\mathrm{AX}+$ trials in groups undergoing unblocking training.
} 
cause $\mathrm{X}$ is a worse predictor of US1 and US2 than is A (i.e., $\left.\lambda-\mathrm{V}_{\mathrm{X}}>\lambda-\mathrm{V}_{\mathrm{A}}\right)$. According to Equation 3, therefore, conditioning to $\mathrm{X}$ will be limited. However, if US2 is omitted on trials with $\mathrm{AX}$ (as was the case for the Down groups) then, because $\mathrm{X}$ enters this stage with no association with US2 $\left(\mathrm{V}_{\mathrm{X}-\mathrm{US} 2}=0\right), \mathrm{X}$ is in fact a better predictor of the absence of US2 than is $\mathrm{A}$ ( as $\mathrm{V}_{\mathrm{A}-\mathrm{US} 2} \approx \lambda$ ). This will offset the reduction in attention to $X$ that results from it being a worse predictor of US1 than A, and, therefore, permit X to better enter into an association with US1 in the Down group than in the Blocking group.

Deriving a prediction from Mackintosh's theory for the Partial group is more complex. This follows because on the $\mathrm{AX}++$ trials $\mathrm{X}$ will be a worse predictor of US2 than A (resulting in a loss of attention to $\mathrm{X}$ ), but on the $\mathrm{AX}+$ trials $\mathrm{X}$ will be a better predictor of the absence of US2 than A (resulting in a gain in attention to X). If the gains and losses in attention to $\mathrm{X}$ change at exactly the same rate, then one might imagine that Mackintosh's theory will predict that attention to $\mathrm{X}$ will oscillate around a level that is somewhere between the level of attention to $X$ in the Blocking and Down groups. And, on this basis therefore, we might anticipate a moderate level of conditioning, but not enhanced unblocking. However, this analysis ignores the fact that, for animals in the Partial group, half of the trials with $\mathrm{X}$ are paired with two USs, and for the Down group none of the trials with $\mathrm{X}$ are paired with two USs. This feature of the design alone may permit Mackintosh's theory to predict superior conditioning to $\mathrm{X}$ in the Partial group relative to the Down group. Of course, appealing to differences in the number of USs that X is paired with will not permit Mackintosh's theory to explain why conditioned responding to $\mathrm{X}$ was greater in the Down group relative to the Blocking group. In order to predict the full pattern of results from Experiment 2, therefore, it seems necessary for Mackintosh's theory to take into account both the associability of $\mathrm{X}$ and the number of USs present on AX trials when determining the ultimate level of conditioned responding to $\mathrm{X}$. This would seem to be an entirely reasonable assumption.

In contrast to the preceding two analyses of unblocking, that emphasize the contribution of variations in the processing of the CS, Holland and Kenmuir (2005) have provided an analysis in terms of variations in the processing of the US. Holland and Kenmuir suggested that the surprising omission of the second US on AX trials may enhance the processing of the first US, thus enabling it to serve as a more effective reinforcer for conditioning. For example, during Stage 1 trials with $\mathrm{A}++$ an association may form between US1 and US2. Analogous to the way in which the associability of a CS declines when it reliably signals a US (Hall $\&$ Pearce, 1979) the processing of US1 might also decline across $\mathrm{A}++$ trials. The omission of US2 on $\mathrm{AX}+$ trials, however, can be expected to extinguish the association between US1 and US2, permitting a restoration in the processing of (the now less reliable) US1, and superior conditioning to any CSs that precede it. Of course, such a treatment will, ultimately, result in the absence of US2 becoming expected on the basis of US1 - once again rendering US1 as a good predictor of the events that follow it, and thus, reducing its processing. It, therefore, follows from this analysis that any manipulation that serves to sustain US1 as an unreliable predictor of US2 might also enhance the processing of US1, in the same way that the associability of a CS can be enhanced by establishing it as an unreliable predictor of the US (e.g., Haselgrove, Esber, Pearce, \& Jones, 2010). This analysis, therefore, seems to provide a natural explanation for the enhancement of unblocking observed in the Partial group of Experiment 2. Where this analysis encounters difficulty with the present data, however, is with the fact that every experimental group in each stage of the experiments received trials in which US1 was followed by US2, and an equal number of trials in which US1 was followed by nothing. There is, therefore, no basis for expecting the omission of US2 in the Down group to enhance the processing of US1, as trials with B in Stage 1 had already arranged for US1 to be followed by the absence of US2. In order to reconcile this analysis with the current results, it is possible to make the additional assumption that some or all components of the CS are configured with US1. In this way, then, the omission of US2 on AX + trials in Stage 2 for the Down groups would constitute a surprising event as US1 following AX is a qualitatively different event to US1 following B. It remains to be determined whether this additional assumption can be justified.

It may be possible to develop an explanation for the results of Experiment 1 in terms of generalization decrement if it is assumed that switching the US on $\mathrm{A}++$ and $\mathrm{B}+$ trials during Stage 1 to $\mathrm{AX}+$ and $\mathrm{B}++$ in Stage 2 in the Down group generates a context change that will not be experienced by animals in the Blocking group. For the Down group, such a context change could result in a concomitant chance in the encoding of $\mathrm{A}$. The resulting generalization decrement would then permit the acquisition of associative strength to $\mathrm{X}$ on $\mathrm{AX}+$ trials for the same reasons as provided by Neely and Wagner (1974). However, the same analysis would seem to encounter difficulty when explaining the superior responding to $\mathrm{X}$ in the Partial group relative to the Down group in Experiment 2. For this group only half the trials were switched from $\mathrm{A}++$ to $\mathrm{AX}+$ and from $\mathrm{B}+$ to $\mathrm{B}++$ between Stage 1 and Stage 2; the remaining trials were consistent with Stage 1 training $(\mathrm{AX}++, \mathrm{B}+)$. Consequently, the context change, and potential for a concomitant change in the encoding of $\mathrm{A}$ is reduced. The more modest generalization decrement in the Partial group, relative to the Down group, would then permit the acquisition of less associative strength to $\mathrm{X}$, in the Partial group than in the Down group, not more.

Our final discussion concerns the analysis of unblocking provided by Rescorla and Colwill (1983). Recall they suggested that the enhanced response to $\mathrm{X}$ following $\mathrm{A}++, \mathrm{AX}+$ training may be due to the formation of an association between $\mathrm{X}$ and $\mathrm{A}$ on the $\mathrm{AX}$ trials. Consequently, at test, $\mathrm{X}$ is able to evoke a strong $\mathrm{CR}$ as it can activate, through the associative chain $\mathrm{X} \rightarrow \mathrm{A} \rightarrow++$, a representation of a stronger US than in control groups for whom Stage 1 training comprised A+. As Rescorla and Colwill note, this analysis requires further assumptions to explain cases of unblocking in which the Down and Blocking groups receive training of the form $\mathrm{A}++$ then $\mathrm{AX}+$, and $\mathrm{A}++$ then $\mathrm{AX}++$, respectively. What might these further assumptions be? One possibility is to suggest that presentation of the US interferes with the establishment of the within-compound association between $\mathrm{X}$ and $\mathrm{A}$; and that furthermore, two USs interfere more with the acquisition of the $\mathrm{X}-\mathrm{A}$ association than one US. If this were the case, then the first link in the $\mathrm{X} \rightarrow \mathrm{A} \rightarrow++$ associative chain may be better established by the Down groups than the Blocking groups. If this were the case, however, then it is difficult to see why responding was higher in the Partial than the Down group in Experiment 2. The Partial group received half of the trials with $\mathrm{AX}++$ and the 
remainder with $\mathrm{AX}+$. On the basis of the previous analysis, therefore, we might imagine responding should lie between the Blocking and Down groups. Alternatively, within compound associations might be particularly prone to establishment when the US is very surprising. Under these circumstances, then, the $\mathrm{X}-\mathrm{A}$ association would be weakest in the Blocking groups (for whom the USs are expected), somewhat less weak in the Down groups (at least until the omitted second US is rendered predictable) and strongest in the Partial group of Experiment 2 (for whom the post-trial US is always surprising). Such an assumption would also provide a natural explanation for why $\mathrm{A}+, \mathrm{AX}-$ at first establishes $\mathrm{X}$ as a second-order CS (when the absence of the US is surprising) but with extended training establishes $\mathrm{X}$ as a conditioned inhibitor (e.g., Rescorla, 1980, p. 10). However, by this token it is difficult to see how within-compound associations would ever form in blocking procedures, despite evidence that they do (Speers, Gillan, \& Rescorla, 1980). Furthermore, this analysis would be inconsistent with the most direct analysis of this issue conducted by Holland (1980) which showed that presentation of a surprising US interfered more with second order conditioning than did a predicted US, not less.

A complete analysis of unblocking by US omission still eludes us. It seems most likely to us that post-trial surprise enhances the processing of the stimuli that precede it. Whether the US, the CS, or both of these stimuli enjoy an enhancement in processing remains to be determined. Furthermore, the mechanism, or mechanisms, that underpin this enhancement in processing have yet to be fully resolved. In any case, the current results place further constraints upon theories of unblocking, as well as demonstrating that the effect can be enhanced under conditions of sustained post-trial surprise.

\section{References}

Beckers, T., Miller, R. R., De Houwer, J., \& Urushihara, K. (2006). Reasoning rats: Forward blocking in Pavlovian animal conditioning is sensitive to constraints of causal inference. Journal of Experimental Psychology: General, 135, 92-102. doi:10.1037/0096-3445.135.1.92

Blaser, R. E., Couvillon, P. A., \& Bitterman, M. E. (2004). Backward blocking in honeybees. Quarterly Journal of Experimental Psychology, 57, 349-360.

Bucci, D. J., Holland, P. C., \& Gallagher, M. (1998). Removal of cholinergic input to rat posterior cortex disrupts incremental processing of conditioned stimuli. The Journal of Neuroscience, 18, 8038-8046.

Cotton, M. M., Goodall, G., Mackintosh, N. J. (1982). Inhibitory conditioning resulting from a reduction in the magnitude of reinforcement. Quarterly Journal of Experimental Psychology, 34, 163-180.

Dickinson, A. (1980). The US-omission effect and static-cue conditioning: A comment on Kremer, Specht, and Allen. Animal Learning \& Behavior, 8, 686-688. doi:10.3758/BF03197787

Dickinson, A., Hall, G., \& Mackintosh, N. J. (1976). Surprise and the attenuation of blocking. Journal of Experimental Psychology: Animal Behavior Processes, 2, 313-322. doi:10.1037/0097-7403.2.4.313

Dickinson, A., Mackintosh, N. J. (1979). Reinforcer specificity in the enhancement of conditioning by post-trial surprise. Journal of Experimental Psychology: Animal Behavior Processes, 5, 162-177. doi: 10.1037/0097-7403.5.2.162

Dopson, J. C., Pearce, J. M., \& Haselgrove, M. (2009). Failure of retrospective revaluation to influence blocking. Journal of Experimental Psychology: Animal Behavior Processes, 35, 473-484. doi:10.1037/ a0014907
Feldman, J. M. (1971). Added cue control as a function of reinforcement predictability. Journal of Experimental Psychology, 91, 318-325. doi: 10.1037/h0031888

Hall, G., \& Pearce, J. M. (1979). Latent inhibition of a CS during CS-US pairings. Journal of Experimental Psychology: Animal Behavior Processes, 5, 31-42. doi:10.1037/0097-7403.5.1.31

Haselgrove, M., Esber, G. R., Pearce, J. M., \& Jones, P. M. (2010). Two kinds of attention in Pavlovian conditioning: Evidence for a hybrid model of learning. Journal of Experimental Psychology: Animal Behavior Processes, 36, 456-470. doi:10.1037/a0018528

Haselgrove, M., \& Evans, L. H. (2010). Variations in selective and nonselective prediction error with the negative dimension of schizotypy. Quarterly Journal of Experimental Psychology, 63, 1127-1149. doi: 10.1080/17470210903229979

Holland, P. C. (1980). Second-order conditioning with and without unconditioned stimulus presentation. Journal of Experimental Psychology: Animal Behavior Processes, 6, 238-250. doi:10.1037/0097-7403.6.3 .238

Holland, P. C. (1984). Unblocking in Pavlovian appetitive conditioning. Journal of Experimental Psychology: Animal Behavior Processes, 10, 476-497. doi:10.1037/0097-7403.10.4.476

Holland, P. C. (1985). Pretraining a compound conditioned stimulus reduces unblocking. Bulletin of the Psychonomic Society, 23, 237-240.

Holland, P. C. (1988). Excitation and inhibition in unblocking. Journal of Experimental Psychology: Animal Behavior Processes, 14, 261-279. doi:10.1037/0097-7403.14.3.261

Holland, P. C., \& Gallagher, M. (1993). Effects of amygdala central nucleus lesions on blocking and unblocking. Behavioral Neuroscience, 107, 235-245. doi:10.1037/0735-7044.107.2.235

Holland, P. C., \& Kenmuir, C. (2005). Variations in Unconditioned stimulus processing in unblocking. Journal of Experimental Psychology: Animal Behavior Processes, 31, 155-171. doi:10.1037/0097-7403.31.2 .155

Kamin, L. J. (1968). “Attention-like” processes in classical conditioning. In M. R. Jones (Ed.), Miami symposium on the prediction of behavior: Aversive stimulation (pp. 9-31). Miami, FL: Appleton-Centaury-Crofts.

Kamin, L. J. (1969). Selective association and conditioning. In N. J. Mackintosh \& W. K. Honig (Eds.), Fundamental Issues in Associative Learning (pp. 42-64). Halifax, Canada: Dalhousie University Press.

Khallad, Y., \& Moore, J. (1996). Blocking, unblocking and overexpectation in autoshaping with pigeons. Journal of the Experimental Analysis of Behavior, 65, 575-591. doi:10.1901/jeab.1996.65-575

Kremer, E. F. (1978). The Rescorla-Wagner model: Losses of associative strength in compound conditioned stimuli. Journal of Experimental Psychology: Animal Behavior Processes, 4, 22-36. doi:10.1037/00977403.4.1.22

Kremer, E. F., Spect, T., \& Allen, R. (1980). Attenuation of blocking with the omission of a delayed US. Animal Learning \& Behavior, 8, 609 616. doi:10.3758/BF03197776

Le Pelley, M. E., Haselgrove, M., \& Esber, G. R. (2012). Modeling attention in associative learning: Two processes or one? Learning \& Behavior, 40, 292-304. doi:10.3758/s13420-012-0084-4

Le Pelley, M. E., Oakeshott, S. M., \& McLaren, I. P. L. (2005). Blocking and unblocking in human causal reasoning. Journal of Experimental Psychology: Animal Behavior Processes, 31, 56-70. doi:10.1037/00977403.31.1.56

Mackintosh, N. J. (1975). A theory of attention: Variations in the associability of stimuli with reinforcement. Psychological Review, 82, 276298. doi:10.1037/h0076778

Mackintosh, N. J., Bygrave, D. J., \& Picton, B. M. B. (1977). Locus of the effect of a surprising reinforcer in the attenuation of blocking. Quarterly Journal of Experimental Psychology, 29, 327-336. doi:10.1080/ 14640747708400608

Mackintosh, N. J., \& Cotton, M. M. (1985). Conditioned inhibition from 
reinforcement reduction. In R. R. Miller \& N. E. Spear (Eds.), Information processing in animals: Conditioned inhibition (pp. 89-111). Hillsdale, NJ: Erlbaum, Inc.

Mackintosh, N. J., Dickinson, A., \& Cotton, M. M. (1980). Surprise and blocking: Effects of the number of compound trials. Animal Learning \& Behavior, 8, 387-391. doi:10.3758/BF03199622

Neely, J. H., \& Wagner, A. R. (1974). Attenuation of blocking with shifts in reward: The involvement of schedule-generated contextual cues. Journal of Experimental Psychology, 102, 751-763. doi:10.1037/ h0036410

Pearce, J. M., \& Hall, G. (1980). A model for Pavlovian conditioning: Variations in the effectiveness of conditioned but not of unconditioned stimuli. Psychological Review, 87, 532-552. doi:10.1037/0033-295X.87 .6 .532

Pearce, J. M., Kaye, H., \& Hall, G. (1982). Predictive accuracy and stimulus associability: Development of a model for Pavlovian conditioning. In M. L. Commons, R. J. Herrnstein, \& A. R. Wagner (Eds.), Quantitative analyses of behavior: Acquisition (Vol. 3, pp. 241-255). Cambridge, MA: Ballinger.

Prados, J., Alvarez, B., Howarth, J., Stewart, K., Gibson, C. L., Hutchinson, C. V., . . Davidson, C. (2012). Cue competition effects in the planarian. Animal Cognition. Advanced online publication. doi:10.1007/ s10071-012-0561-3

Rescorla, R. A. (1980). Pavlovian second order conditioning: Studies in associative learning. Hillsdale, $\mathrm{NJ}$ : Erlbaum.

Rescorla, R. A., Colwill, R. M. (1983). Within-compound associations in unblocking. Journal of Experimental Psychology: Animal Behavior Processes, 9, 390-400. doi:10.1037/0097-7403.9.4.390
Rescorla, R. A., \& Wagner, A. R. (1972). A theory of Pavlovian conditioning: Variations in the effectiveness of reinforcement and nonreinforcement. In A. H. Black \& W. K. Prokasy (Eds.), Classical conditioning (Vol. 2, pp. 64-99). New York, NY: Appleton-Century-Crofts.

Ross, R. T. (1985). Blocking and unblocking of conditioned analgesia. Learning and Motivation, 16, 173-189. doi:10.1016/00239690(85)90011-6

Schultz, W., \& Dickinson, A. (2000). Neuronal coding of prediction errors. Annual Review of Neuroscience, 23, 473-500. doi:10.1146/annurev .neuro.23.1.473

Speers, M. A., Gillan, D. J., \& Rescorla, R. A. (1980). Within-compound associations in a variety of compound conditioning procedures. Learning and Motivation, 11, 135-149. doi:10.1016/0023-9690(80)90009-0

Tennant, W. A., \& Bitterman, M. E. (1975). Blocking and overshadowing in two species of fish. Journal of Experimental Psychology: Animal Behavior Processes, 1, 22-29. doi:10.1037/0097-7403.1.1.22

Waelti, P., Dickinson, A., \& Schultz, W. (2001). Dopamine responses comply with basic assumptions of formal learning theory. Nature, 412, 43-48. doi:10.1038/35083500

Wagner, A. R., Mazur, J. E., Donegan, N. H., \& Pfautz, P. L. (1980), Evaluation of blocking and conditioned inhibition to a CS signaling a decrease in US intensity. Journal of Experimental Psychology: Animal Behavior Processes, 6, 376-385. doi:10.1037/0097-7403.6.4.376

Received November 2, 2012

Revision received March 4, 2013

Accepted March 8, 2013

\section{E-Mail Notification of Your Latest Issue Online!}

Would you like to know when the next issue of your favorite APA journal will be available online? This service is now available to you. Sign up at http://notify.apa.org/ and you will be notified by e-mail when issues of interest to you become available! 